\title{
Idiopathic cerebellar ataxia of late onset: natural history and MRI morphology
}

\author{
T Klockgether, G Schroth, H-C Diener, J Dichgans
}

\begin{abstract}
Twenty eight patients with the clinical diagnosis of idiopathic late onset cerebellar ataxia were examined clinically and by magnetic resonance imaging (MRI) or computed tomography (CT). In addition, the clinical records of all patients were analysed retrospectively. On the basis of their clinical presentation they were subdivided into patients with a pure cerebellar syndrome $(n=9)$ and patients with a cerebellar syndrome and additional non-cerebellar symptoms ( $=13$ ). No attempts were made to classify patients with a disease duration of less than four years $(n=6)$ because the retrospective analysis showed that the disease started almost invariably with a pure cerebellar syndrome and additional symptoms came later. Patients with a lasting pure cerebellar syndrome had a significantly better prognosis than patients with additional non-cerebellar involvement (annual progression rate rate: $0 \cdot 40$ versus $0 \cdot 80$ ). Calculated median lifetime from onset of symptoms was $\mathbf{2 0 . 7}$ years in patients with a pure cerebellar syndrome and $7 \cdot 7$ years in patients with additional non-cerebellar symptoms. Among the latter, disease progression was faster the earlier non-cerebellar symptoms occurred. All of them presented with Parkinsonian symptoms, whereas bulbar symptoms, vertical gaze paresis, pyramidal deficits, dementia and urinary incontinence were encountered less frequently. MRI or CT showed cerebellar atrophy without apparent involvement of brainstem structures in all patients with a pure cerebellar syndrome suggesting the diagnosis of cerebellar cortical atrophy (CA). The majority of the patients with additional non-cerebellar symptoms had evidence of an atrophy of the cerebellum and the brainstem suggesting the presence of olivo-ponto-cerebellar atrophy (OPCA). In two of them, however, MRI morphology was not compatible with the diagnosis of OPCA.
\end{abstract}

The term idiopathic cerebellar ataxia of late onset which was first introduced by Harding ${ }^{1}$ refers to a group of sporadically occurring degenerative diseases of the cerebellum and the brainstem with unknown origin. The idiopathic label distinguishes them from symp- tomatic cerebellar ataxias which may occur due to alcoholism, hypothyroidism, inflammation, malignancy and other causes. ${ }^{23}$ Similar to hereditary cerebellar ataxias the neuropathological changes found in brains at necropsy are heterogenous. ${ }^{3-7}$ In cases of cerebellar cortical atrophy (CA) the degenerative process mainly affects the Purkinje cells of the cerebellar cortex with facultative retrograde cell loss of minor degree in the inferior olives. According to Greenfield, ${ }^{2}$ these cases are neuropathologically almost identical to Holmes ${ }^{8}$ hereditary cases of cerebello-olivary atrophy. In many cases the atrophy is most severe in the upper vermis and anterior part of the cerebellum, as first described by Marie et $a l^{9}$ and termed late cerebellar cortical atrophy. In contrast, in cases of olivo-ponto-cerebellar atrophy (OPCA $)^{10}$ the pathological process is thought to start in the olivary and pontine nuclei with transneuronal changes mainly in the cerebellar hemispheres. In association with OPCA pathological changes have been described in various parts of the central nervous system, including the spinal cord, thalamus, basal ganglia and cerebral cortex. The widespread nature of the neuropathological changes seen in the brains of some patients has led some authors to classify these cases as multiple system atrophies. ${ }^{11}$

Although clinicians tend to assume that patients with cerebellar cortical atrophy have a relatively pure cerebellar syndrome on clinical examination and have a better prognosis than those with OPCA, this view has been discredited by a number of studies. Zülch ${ }^{12}$ reported a series of 34 necropsy cases with pure cerebellar atrophy, seven of which had extrapyramidal symptoms during life. Similarly, Eadie $^{13}$ collected numerous reports of families with necropsy-proven cerebello-olivary atrophy in which non-cerebellar symptoms, such as dementia, bladder dysfunction, Parkinsonian symptoms and spasticity had been present during life. Conversely, Destunis ${ }^{14}$ described a patient with a pure cerebellar syndrome, who had OPCA at necropsy. The fact that the neuropathological picture of OPCA and CA may occur in different members of the same family ${ }^{15}$ raises further doubt that a classification based on morphological criteria is clinically useful. Nevertheless, with the introduction of modern neuroimaging techniques, such as computed tomography (CT) and nuclear magnetic resonance imaging (MRI), the visualisation of infratentorial structures has become possible during life and radiological criteria to distinguish between pure cerebellar atrophy and OPCA have been elaborated. ${ }^{16-21}$
Received 17 May 1989 and in revised form 19 July 1989. 
In an alternative approach Harding ${ }^{122}$ used clinical and genealogical criteria to classify the hereditary and idiopathic ataxias of late onset. In her study on idiopathic cerebellar ataxia of late onset Harding ${ }^{1}$ distinguished between patients clinically corresponding to those generally labelled as CA (group A) and patients clinically corresponding more or less to those with sporadic OPCA (group C). A smaller group comprised patients with distinct intentional tremor, some of whom might have been diagnosed by others as dyssynergia cerebellaris myoclonica or essential tremor. In Harding's ${ }^{1}$ patients radiological and neuropathological data were available only to a limited degree so that it remains open, if the assumptions about the correspondence of Harding's groups to known neuropathological categories are correct.

To help to resolve the numerous controversies surrounding the degenerative diseases of the cerebellum, there is an apparent need for studies in which detailed clinical and morphological data of a sufficiently large number of patients are compared. This study describes the natural history and MRI morphology of patients with idiopathic late onset ataxia. A clinical classification based on the differentiation betwen patients with a pure cerebellar syndrome and patients with additional noncerebellar symptoms is proposed and contrasted with a classification of the same patients on the basis of morphological criteria, as defined by MRI. It is the aim of the present study to see 1 ) to what extent clinically defined subgroups correspond to the known morphological categories and 2) whether clinical criteria of prognostic relevance can be identified.

\section{Methods}

Twenty eight patients referred to our department between 1983-87 were included in this study. All fulfilled the following criteria: 1) Progressive cerebellar ataxia with an onset after the age of 20 years and without preceding neurological symptoms. 2) No evidence of a focal cerebellar disease, such as tumour, ischaemia or haemorrhage in CT and/or MRI. 3) Absence of any similar disorder in relatives and no evidence of consanguinity of their parents. 4) No evidence for a non-focal, symptomatic origin of the disorder. To make sure that symptomatic forms of cerebellar ataxia were excluded, patients with a history of alcoholism, malignancy, chronic diarrhoea, significant unexplained weight loss and chronic intake of anticonvulsant medication did not enter into this study. In addition, patients with evidence of hypothyroidism or vitamin Edeficiency were excluded. The cerebrospinal fluid was examined in all patients to identify patients with multiple sclerosis and other inflammatory diseases of the central nervous system.

All patients were clinically examined by one of us (TK) using a standardised examination procedure. Severity of cerebellar symptoms was rated on a scale ranging from zero (absent)

\section{Table 1 Scaling of cerebellar symptoms}

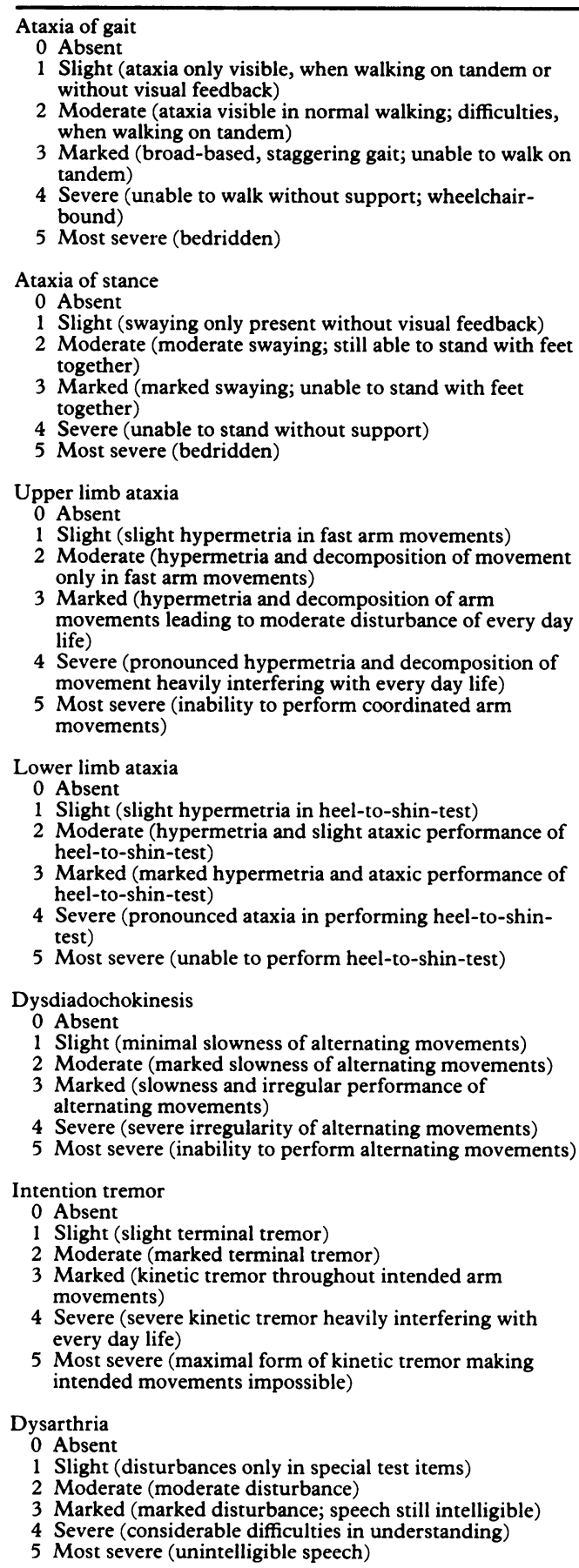

to five (most severe). The criteria used for scaling of cerebellar symptoms are given in table 1 . The annual progression rate was calculated by dividing the rated severity of gait ataxia by the disease duration in years. Patients who died of the disease were given a score of $\operatorname{six}^{24}$

On the day of the clinical examination MRI was performed using a superconducting system operating at $1.5 \mathrm{~T}$ field strength (Magnetom, Siemens AG, Erlangen, West Germany) with a head coil of $30 \mathrm{~cm}$ diameter. Data were acquired and displayed on a $256 \times 256$ matrix. A standard examination programme was used which included sagittal and axial T1-weighted images without gap (spin echo, TR $=600 \mathrm{~ms}$, $\mathrm{TE}=22 \mathrm{~ms}$, slice thickness $=4 \mathrm{~mm}$, two averages). The first axial slice was at the 
intervertrebral disc space $\mathrm{C} 2 / \mathrm{C} 3$, the last was situated apical to the lateral ventricles. In addition, three slices (mediosagittal and $8 \mathrm{~mm}$ parasagittal, left and right) with a CPGM (Carr-Purcell-Gill-Meibom) sequence were taken and T2-relaxation times were calculated from these echoes by linear regression. Severity of atrophic changes of the cerebellar hemispheres, the upper vermal region, the lower vermal region, the brainstem at the level of medulla oblongata and the pons and enlargement of the fourth ventricle were rated by two independent experienced examiners (not aware of the clinical findings) and using a score ranging from zero (normal) to three (severe). For comparison the MRIs of 15 age-matched healthy control subjects were rated together with those of patients. Seven patients in whom MRI could not be performed due to their poor clinical condition $(n=3)$, metallic implants $(n=2)$ or claustrophobia $(n=2)$ received a high-resolution CT. The CTs were performed on a third generation scanner (Somatom DRH, Siemens AG, Erlangen, West Germany) with a $512 \times 512$ matrix.

In addition, the clinical records of all patients were analysed retrospectively. All patients had been clinically examined by different investigators in our department at least once in two years. A standard examination procedure identical with that mentioned above was used routinely.

\section{Results}

There were 28 patients eight of whom died during completion of the study. Twelve patients were female and 16 male. The mean age at the onset of symptoms was $54 \cdot 2(8 \cdot 8)$ years (range: $38-70$ years). At the time of the last clinical examination the mean duration of symptoms was $6 \cdot 1(4 \cdot 7)$ years. In the deceased patients the duration of the disease ranged between four and 25 years (mean: 8.9, 6.6

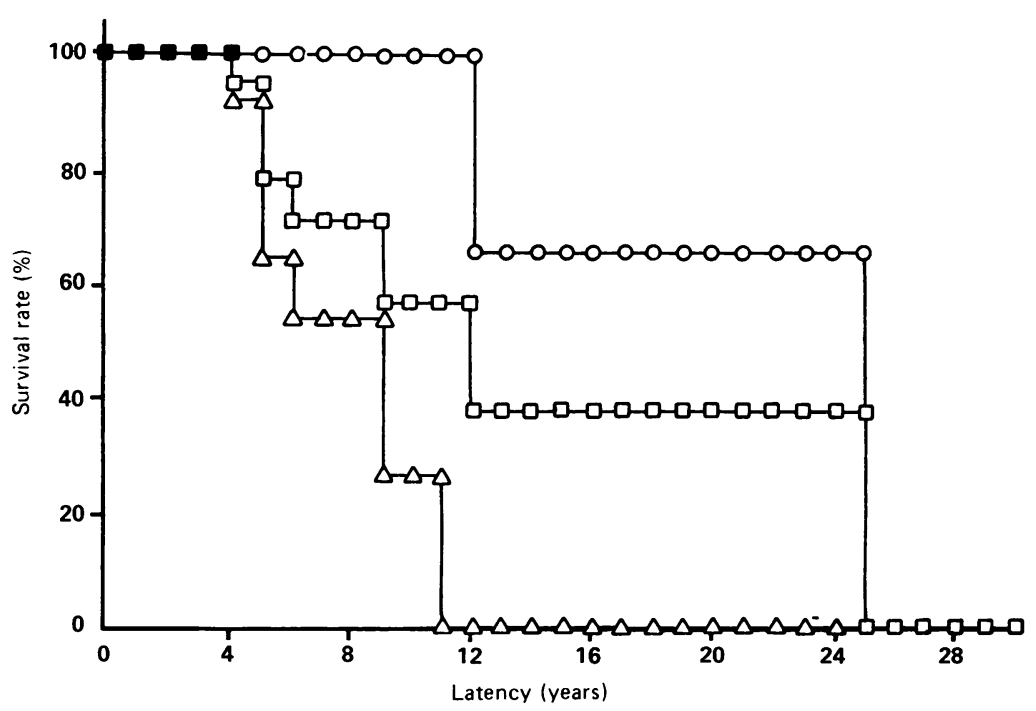

Figure 1 Survival function of patients with idiopathic late onset cerebellar ataxia, as calculated by the Kaplan-Meier life-table method. ${ }^{25}$ Ordinate: percentage of patients surviving; abscissa: latency from onset of symptoms. Squares represent the entire sample $(n=28)$ (median lifetime from onset of symptoms: 14.5 years), circles patients with a pure cerebellar syndrome $(n=9)$ (median lifetime: $20 \cdot 7$ years) and diamonds patients with additional non-cerebellar symptoms $(n=13)$ (median lifetime: $7 \cdot 7$ years). years). Fig 1 shows the survival function, as calculated by the Kaplan-Meier life-table method. ${ }^{25}$ Median lifetime of the entire sample was estimated as 14.5 years.

Fifteen patients had an almost pure cerebellar syndrome whereas the remaining ones presented with additional non-cerebellar symptoms. A retrospective analysis revealed a steadily increasing percentage of patients with additional non-cerebellar symptoms. Whereas at the onset of the disease only a minority of patients had additional non-cerebellar symptoms (two out of 28 patients), the percentage increased to $52.6 \%$ (10 out of 19 patients) after a duration of five years. Later the proportion decreased again reflecting the fact that the patients with a pure cerebellar syndrome had a longer life expectancy. The mean latency until the first occurrence of non-cerebellar symptoms was $3.4(1.8)$ years.

For these reasons we decided to treat patients with a disease duration of less than four years as a separate group (group $1, \mathrm{n}=6$ ). Among the patients with a disease duration of four years or more nine patients had an almost pure cerebellar syndrome (group 2), whereas the remaining 13 had additional non-cerebellar symptoms (group 3 ).

\section{Patients with a disease duration of less than}

four years (group 1 )

All six patients with a disease duration of less than four years presented with a rather uniform clinical picture. Their mean age of onset was $50.5(6.4)$ years. There were no significant differences between the results of the initial clinical examination, $1.5(0.8)$ years, and those of the final one, $2.0(0.8)$ years after the onset of symptoms (table 2 ). They had moderate ataxia of stance and gait and marked ataxia of the lower extremities, whereas the upper extremities were less affected. There was slight dysdiadochokinesis, whereas intention tremor was present in only one out of six patients. Four out of six patients had dysarthria. Clinical examination revealed mild to marked cerebellar oculomotor abnormalities in all patients. Nystagmus on lateral gaze and pursuit-related deficits with impaired smooth pursuit, reduced optokinetic nystagmus and incomplete suppression of the vestibulo-ocular reflex by fixation of a stationary target were the most frequent findings.

\section{Patients with a pure cerebellar syndrome (group 2)}

In nine patients who had been followed up for at least four years a pure cerebellar syndrome was encountered. The age at the onset of symptoms in this group was $53 \cdot 1(10 \cdot 2)$ years. At the time of the initial clinical examination, $3.3(3.0)$ years after the onset of symptoms, ataxia of gait and stance was present in all nine patients, dysarthria in six, upper limb ataxia in three and lower limb ataxia in four. Intention tremor was not reported in any of these patients (table 2).

In the later course of the disease the cerebellar syndrome became more pronounced, but the annual progression rate remained 
Table 2 Incidence and severity of cerebellar symptoms. Patients of group 1 (disease duration of less than four years) $(n=6)$ were examined $1 \cdot 5$ $(0.8)$ years (initial examination) and 2.0 (0.8) years (final examination) after the onset of symptoms, patients of group 2 (pure cerebellar syndrome) $(n=9) 3.3(3.0)$ years (initial examination) and 9.5 (5.9) years (final examination) and patients of group 3 (additional noncerebellar symptoms $(n=13) 1 \cdot 7+1 \cdot 1$ years (initial examination) and $5.6(2.4)$ years (final examination) after the onset. The incidence of each symptom is given in percentages. In the final examination the severity was rated on a scale ranging from zero (absent) to five (most severe). The median values and ranges for each group are given. Only group 2 and group 3 were statistically compared. Significances: $\star_{p}<0.05,{ }^{*} p<0.02$ vs. group 2, Mann-Whitney U-test; $\dagger p<0.001$ vs. group 2, chi-square test.

\begin{tabular}{|c|c|c|c|c|c|c|c|c|}
\hline & & $\begin{array}{l}\text { Ataxia } \\
\text { of gait }\end{array}$ & $\begin{array}{l}\text { Ataxia } \\
\text { of stance }\end{array}$ & $\begin{array}{l}\text { Upper } \\
\text { limb ataxia }\end{array}$ & $\begin{array}{l}\text { Lower } \\
\text { limb ataxia }\end{array}$ & $\begin{array}{l}\text { Dysdia- } \\
\text { dochokinesia }\end{array}$ & $\begin{array}{l}\text { Intention } \\
\text { tremor }\end{array}$ & Dysarthria \\
\hline $\begin{array}{l}\text { Group } 1 \mathrm{n}=6 \\
\text { Initial examination } \\
\text { Final examination }\end{array}$ & $\begin{array}{c}\text { Incidence }(\%) \\
\text { Incidence }(\%) \\
\text { Severity (median) } \\
\qquad \text { (range) }\end{array}$ & $\begin{array}{l}100 \\
100 \\
2 \\
2-4\end{array}$ & $\begin{array}{c}83 \\
100 \\
2 \cdot 5 \\
2-3\end{array}$ & $\begin{array}{l}67 \\
83 \\
1 \\
0-3\end{array}$ & $\begin{array}{c}50 \\
100 \\
3 \\
1-3\end{array}$ & $\begin{array}{l}33 \\
83 \\
2 \\
0-3\end{array}$ & $\begin{array}{l}0 \\
17 \\
0 \\
0-3\end{array}$ & $\begin{array}{l}50 \\
67 \\
1 \cdot 5 \\
0-3\end{array}$ \\
\hline $\begin{array}{l}\text { Group } 2 \mathrm{n}=9 \\
\text { Initial examination } \\
\text { Final examination }\end{array}$ & $\begin{array}{l}\text { Incidence }(\%) \\
\text { Incidence }(\%) \\
\text { Severity (median) } \\
\qquad \text { (range) }\end{array}$ & $\begin{array}{c}100 \\
100 \\
3 \\
2-4\end{array}$ & $\begin{array}{r}89 \\
100 \\
3 \\
2-4\end{array}$ & $\begin{array}{c}44 \\
100 \\
2 \\
1-3\end{array}$ & $\begin{array}{c}44 \\
100 \\
3 \\
2-3\end{array}$ & $\begin{array}{l}33 \\
89 \\
3 \\
0-3\end{array}$ & $\begin{array}{c}0 \\
22 \\
0 \\
0-1\end{array}$ & $\begin{array}{l}67 \\
89 \\
3 \\
0-3\end{array}$ \\
\hline $\begin{array}{l}\text { Group } 3 \mathrm{n}=13 \\
\text { Initial examination } \\
\text { Final examination }\end{array}$ & $\begin{array}{c}\text { Incidence }(\%) \\
\text { Incidence }(\%) \\
\text { Severity (median) } \\
\text { (range) }\end{array}$ & $\begin{array}{l}100 \\
100 \\
4^{\star \star} \\
3-5\end{array}$ & $\begin{array}{l}85 \\
100 \\
4^{\star \star} \\
2-5\end{array}$ & $\begin{array}{l}53 \\
100 \\
3 \star \\
2-4\end{array}$ & $\begin{array}{c}77 \\
100 \\
3 \\
2-5\end{array}$ & $\begin{array}{c}54 \\
100 \\
3 \\
2-4\end{array}$ & $\begin{array}{l}8 \\
92 \dagger \\
2 \star \star \\
0-4\end{array}$ & $\begin{array}{c}77 \\
100 \\
3 \cdot 5 \\
1-4\end{array}$ \\
\hline
\end{tabular}

moderate (median: $0 \cdot 40$, range: $0 \cdot 17-0 \cdot 60$ ). At the time of the final clinical examination, 9.5 $(5.9)$ years after the onset of symptoms, all patients had moderate to marked ataxia of gait and stance (table 2). Ataxia of the extremities was now observed in all patients and was more pronounced in the lower limbs than in the upper limbs. Intention tremor was found in only two out of nine patients, whereas a moderate to marked disturbance of diadochokinesis was present in all but one patient. Dysarthria was a common finding (eight out of nine patients), but speech was intelligible in all patients. Cerebellar oculomotor disturbances, such as nystagmus on lateral gaze, impaired smooth pursuit, reduced optokinetic nystagmus, incomplete suppression of the vestibuloocular reflex were found in all patients of this group. Rebound nystagmus and fixation instability were less common findings.

Patients with additional non-cerebellar symptoms (group 3)

This group consisted of 13 patients, all of whom had additional non-cerebellar symptoms. The mean age of onset in this group was $56.7(7 \cdot 8)$ years. Six patients of this group died during the study; the mean disease duration at the time of the final clinical examination was $5 \cdot 6(2 \cdot 4)$ years.

At the time of the initial clinical examination $(1 \cdot 7,1 \cdot 1$ years after the first onset of symptoms) all patients had cerebellar signs (table 2 ). There were only two, in which additional noncerebellar symptoms (akinesia) were present at that time. Among the cerebellar signs ataxia of gait and stance were the most frequent, whereas ataxia of the limbs occurred in about two thirds of the patients and intention tremor was an unusual finding. Ten out of 13 patients had a dysarthric speech disturbance at the time of the initial clinical examination.

In the majority of patients there was a rapid clinical deterioration (median annual progression rate: $0 \cdot 80$, range: $0 \cdot 43-1 \cdot 50)$. In the final clinical examination, $5.6(2.4)$ years after the onset of symptoms, twelve out of 13 patients were wheelchair-bound or bedridden. Limb ataxia was present in all and affected the upper and lower extremity to roughly the same degree. Intention tremor was found in all but one patient. All patients of this group were dysarthric, in seven of them speech was unintelligible for someone who was not familiar with the patient (table 2). Non-cerebellar symptoms appeared in parallel with the worsening of the cerebellar syndrome. The mean latency until the occurrence of the first non-cerebellar symptom was $3.4(1.8)$ years.

Parkinsonian symptoms in varying combinations were present in all patients of this group. Akinesia was observed in eleven, muscular rigidity in nine and resting tremor in seven. Akinesia manifested itself by marked slowness of limb movements, facial impassiveness and an akinetic-shuffling gait in those patients who were not bedridden. Choreiform movements were seen in only one patient. Bulbar dysfunction was present in seven out of 13 patients. Five complained of dysphagia; in two of them dysphagia occurred in combination with affective incontinence and in one patient affective incontinence occurred in the absence of dysphagia. There was no atrophy of the tongue which suggested a supranuclear origin of their bulbar dysfunction. One patient had bilateral paresis of the recurrent nerve. On average, Parkinsonian symptoms occurred earlier $(3 \cdot 5$, $2 \cdot 1$ years after the onset of symptoms) than bulbar signs $(4.4,1.6$ years).

Other symptoms found in patients with Parkinsonian symptoms were dementia of mild degree (three out of 13 patients) and urinary incontinence (five out of 13 patients). There was no apparent association between the occurrence of dementia and that of urinary incontinence. Both symptoms tended to occur later in the course of the disease (dementia: $5 \cdot 3$, 1.2 years, urinary incontinence: $4.8,1.5$ years after the first onset of symptoms) compared with Parkinsonian and bulbar symptoms. Blood pressure in lying or standing position was usually in the lower normal range. None of our patients suffered from overt orthostatic hypotension.

Three patients had hyperreflexia, extensor 
Figure 2 Relationship between the progression rate of the disease and the latency until the

occurrence of the first noncerebellar symptoms in years $(n=13)$. Annual progression rates were calculated by dividing the severity of gait ataxia, rated on a scale from zero (absent) to five (most severe), by the duration of the disease. Patients who died of the disease received a score of six; $r=-0.73$, $p<0.05$, Spearman's rank correlation test.

Figure $3 A: M R I$ of $a$ 56 year old male patient with a pure cerebellar syndrome (disease duration: 18 years). Mediosagittal and axial T1-weighted spin echo images (1.5 T,

$T R=600 \mathrm{~ms}$,

$T E=22 \mathrm{~ms}$ ) show marked atrophy of the cerebellum. The appearance of the brain stem and fourth ventricle is normal.

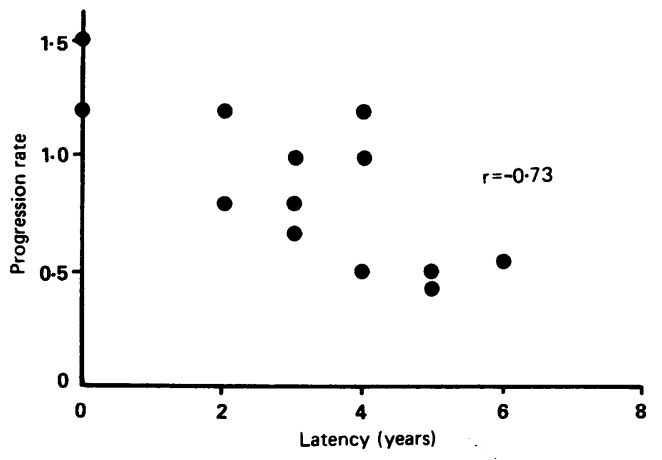

plantar responses and a spastic increase of muscle tone. All of them had additional Parkinsonian and bulbar symptoms. In three other patients hyperreflexia was found without overt spasticity. In all patients cerebellar oculomotor disturbances were observed which included pursuit-related deficits, nystagmus on lateral gaze and reduced optokinetic nystagmus. Three patients had a vertical gaze paresis.

Comparison of groups and prognostic factors There was no statistically significant difference between the age of onset in the three groups. The clinical presentation at the time of the initial clinical examination was rather uniform in all three groups: it was characterised by ataxia of gait and stance, limb ataxia mainly of the legs and to a minor degree, and the rare

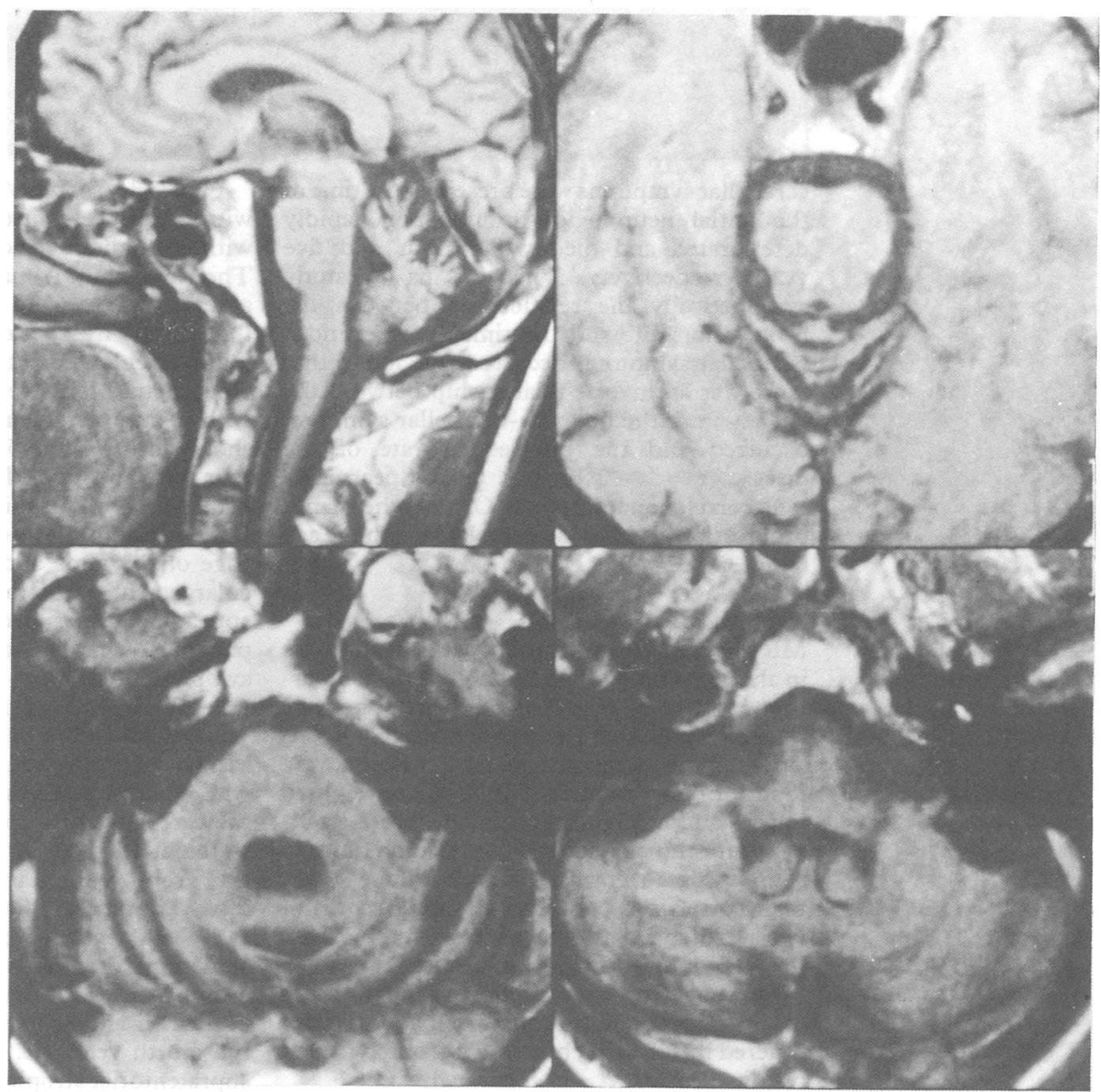

occurrence of intention tremor and dysarthria (table 2). The exceptions were two patients who initially had Parkinsonian symptoms in addition to their cerebellar ataxia. Patients developing non-cerebellar symptoms exhibited a more rapid progression (median annual progression rate: $0 \cdot 80$, range: $0 \cdot 43-1 \cdot 50$ ) than those with a pure cerebellar syndrome (median: $0 \cdot 40$, range: $0 \cdot 17-0 \cdot 60$ ). Although overlap existed between both groups, the difference was statistically highly significant $(\mathrm{p}<0.02$, Mann-Whitney u test).

In the final clinical examination the cerebellar syndrome was more pronounced in patients with additional non-cerebellar symptoms than in those with a pure cerebellar syndrome, although the duration of symptoms was shorter, $5.6(2.4)$ versus $9.5(5.9)$ years (table 2). The most apparent difference was the incidence of intention tremor which was present in only $22 \%$ of the patients in the cerebellar group and in $92 \%$ of the patients in the group with additional non-cerebellar symptoms ( $p<0.001$, chi square test).

A separate life-table analysis for both groups showed that death occurred earlier in patients with additional non-cerebellar symptoms (calculated median lifetime from onset of symptoms: $7 \cdot 7$ years, Kaplan-Meier life-table method) ${ }^{23}$ than in patients with a pure cerebellar syndrome (median lifetime: $20 \cdot 7$ years) (fig 1). Those patients in whom non- 
B: MRI of a 53 year old female patient with cerebellar ataxia and Parkinsonism (disease duration: 4 years) Mediosagittal and axial images demonstrate diffuse cerebellar atrophy, severe shrinkage of the pons and olives and marked enlargement of the fourth ventricle (for examination technique see fig $3 A$ ).

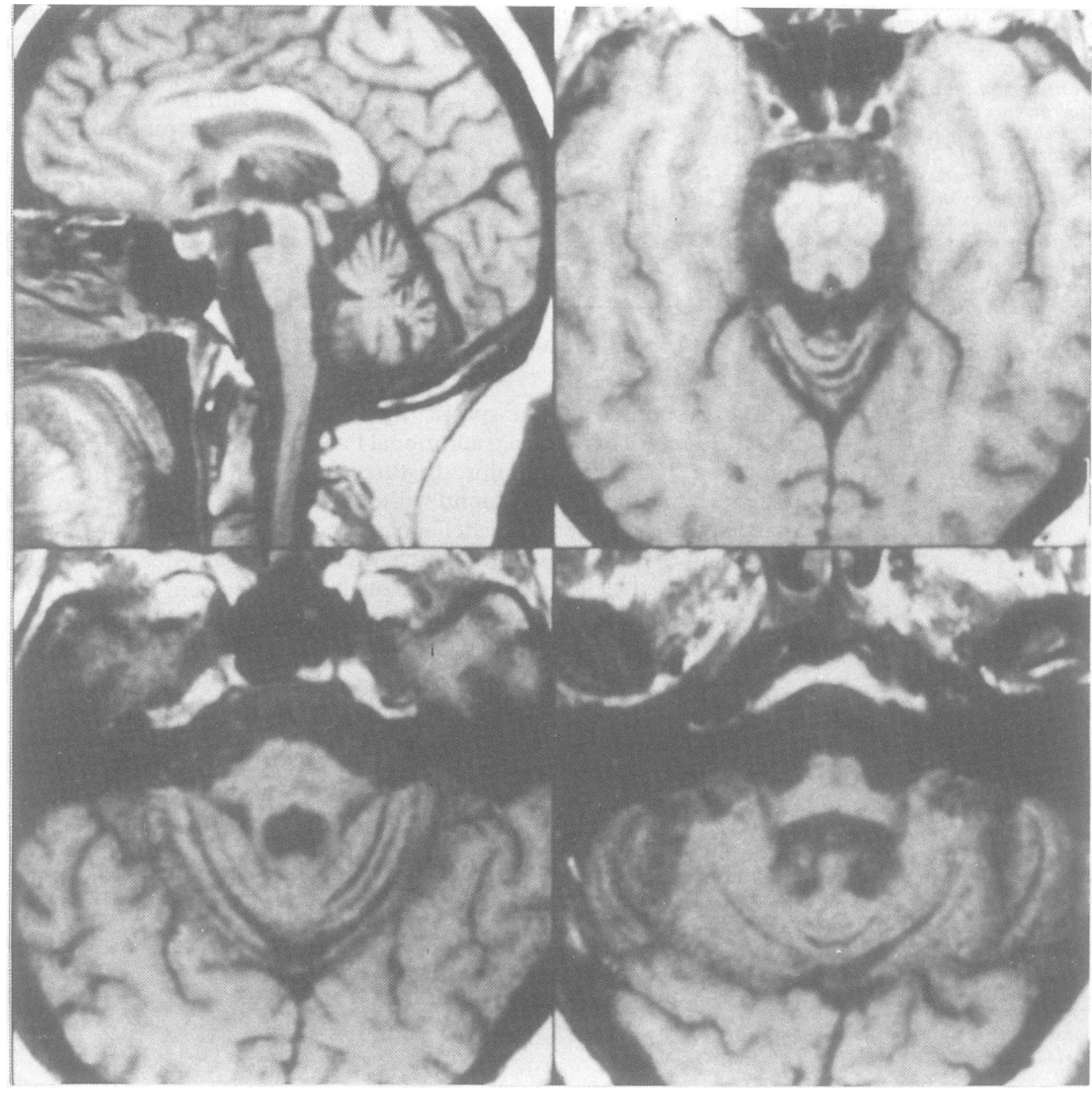

Figure 3A shows MRI of a 56 year old male cerebellar symptoms were present at the time of the initial neurological examination rapidly deteriorated and died within four and five years, respectively. This led us to study systematically the relationship between the progression rate and the time at which noncerebellar symptoms occurred. We found that there was an inverse relationship between the latency until the first non-cerebellar symptom occurred and the progression rate of the disease ( $r=-0.73, p<0.05$, Spearman's rank correlation test) (fig 2). On the other hand, progression rate was not correlated with the age of onset.

\section{Neuroradiological findings}

MRIs were performed in 21 patients and CTs in the remaining seven patients. For comparison MRI scans of 15 healthy control persons were also analysed. In all patients signal intensities of the brainstem and cerebellum were found to be normal on T1- and T2-weighted images and no significant changes were established for the calculated T2-relaxation times in comparison to normal subjects. There was a high correlation between the rating scores of two independent examiners $(r=0.88$, $\mathrm{p}<0.001$, Spearman's rank correlation test). The rating scores were identical in 81 of 126 instances (six rating scores per patient); they differed by 0.5 in 38 , by 1.0 in five and by 1.5 in two instances. with an 18 year history of cerebellar ataxia without additional non-cerebellar symptoms. The cerebellum is markedly atrophic with an accentuation on the upper vermis, whereas there is no shrinkage of the brainstem and only minimal enlargement of the fourth ventricle. This picture is representative for all patients with a pure cerebellar syndrome. Figure 3B shows an MRI of a 53 year old female with a four year history of cerebellar ataxia with additional Parkinsonian symptoms. There is diffuse atrophy of the cerebellum, severe shrinkage of the pons and medulla and a marked enlargement of the fourth ventricle. A similar appearance was noted in the majority of our patients with additional non-cerebellar symptoms (fig 4). There were, however, exceptions. A 70 year old female with a four year history of cerebellar ataxia and a two year history of Parkinsonism presented on MRI with an atrophy of the upper vermis, whereas the cerebellar hemispheres and the brainstem were not affected (fig 3C). In a second case of this group, a 68 year old male patient with a disease duration of four years presenting clinically with severe cerebellar ataxia, dysarthria, akinesia and spasticity had diffuse cerebellar atrophy in MRI without shrinkage of the brainstem and without major enlargement of the fourth ventricle. In addition, a moderate supratentorial atrophy was noted. 
C:MRI of a 70 year old female patient with cerebellar ataxia and Parkinsonism (disease duration: 4 years).

Mediosagittal and axial images demonstrate moderate atrophy of the upper vermis without significant involvement of the brain stem (for examination technique see fig $3 A$ ).

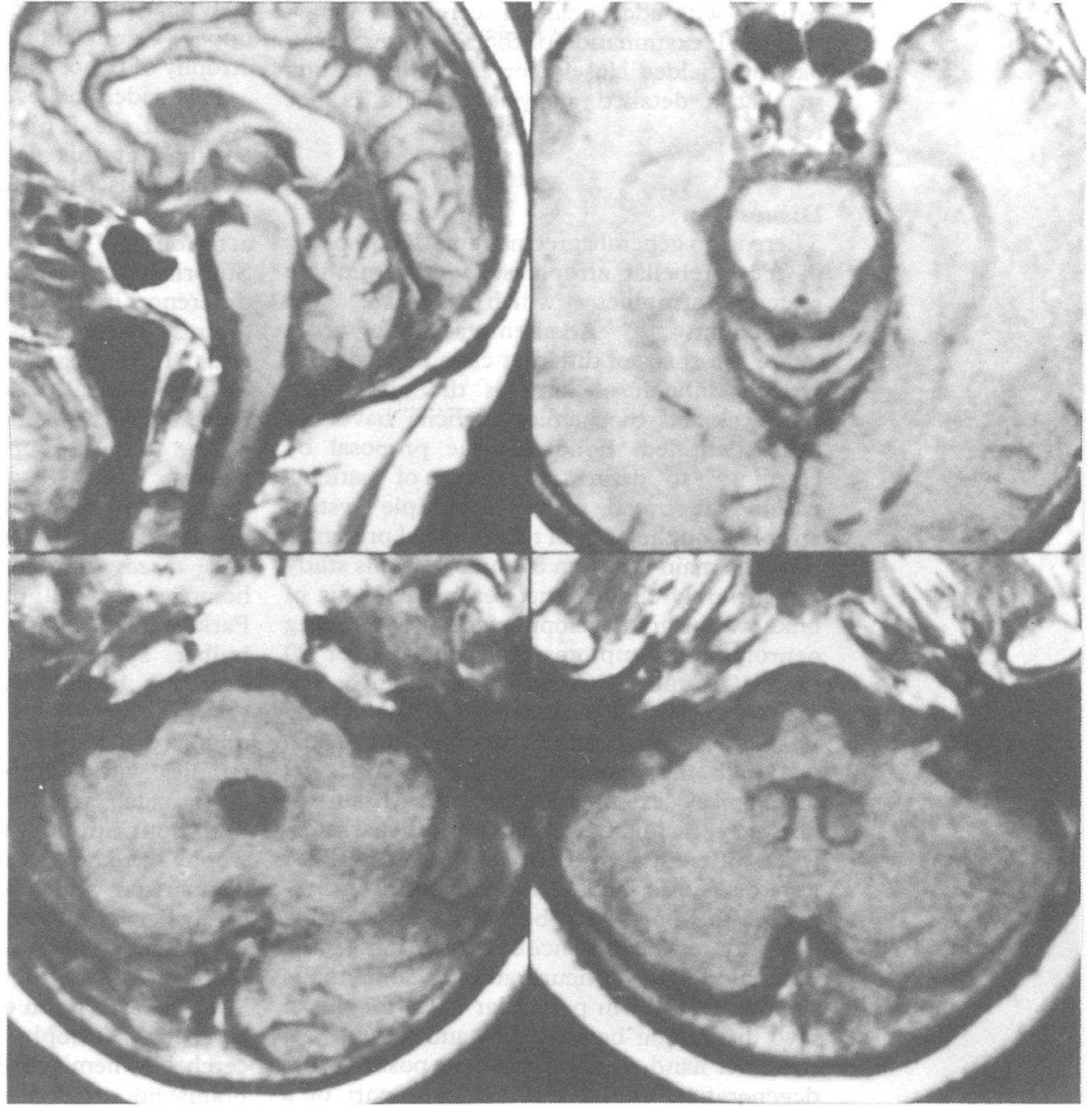

Median rating scores in control subjects $(\mathrm{n}=15)$ were zero for all structures analysed. On average the severity of cerebellar atrophy, as shown on MRI was comparable in patients with a pure cerebellar syndrome $(n=6)$ and those with additional non-cerebellar symptoms

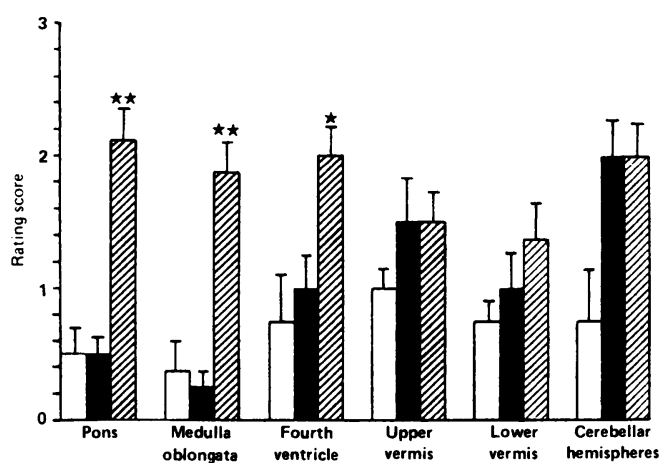

Figure 4 Severity of the atrophy of the brain stem (medulla oblongata, pons), fourth ventricle and cerebellum (upper vermis, lower vermis, hemispheres) in patients with a disease duration of less than four years (group 1 , open bars) ( $n=5)$, patients with a pure cerebellar syndrome (group 2 , filled bars) $(n=6)$ and patients with additional non-cerebellar symptoms (group 3 , hatched bars) ( $n=10)$ as estimated from magnetic resonance imaging (MRI). The degree of atrophy of each structure was rated independently by two experienced examiners who were unaware of the clinical findings using a rating scale ranging from zero (normal) to three (severe). Median values and standard error means are given. Only group 2 and group 3 were statistically compared. Significances: ${ }^{\star} p<0.05,{ }^{\star \star} p<0.02$ vs. group 2, Mann-Whitney U-test.
( $n=10$ ) (fig 4). The appearance of the brainstem, however, differed considerably in both groups. Whereas the medulla oblongata and pons appeared almost normal in all patients with pure cerebellar ataxia (scores of one or less), they were estimated as severely shrunken in patients with additional non-cerebellar symptoms (scores of more than one) with the two exceptions noted above ( $p<0.02$, MannWhitney U-test). In addition, enlargement of the fourth ventricle was more pronounced in patients with additional non-cerebellar symptoms compared with those with a pure cerebellar syndrome $(p<0.05$, MannWhitney U-test). As expected, the MRIs of the patients with a disease duration of less than four years $(n=5)$ showed less severe atrophy. Brainstem structures, fourth ventricle, cerebellar vermis and hemispheres were affected to almost the same degree (fig 4). There was only one patient in this group with marked atrophy of brainstem structures. It remains to be seen if this may be of prognostic relevance. When analysing the distribution of rating scores among all patients, it turned out that the distribution of scores for the atrophy of cerebellar structures was unimodal, whereas the distribution of scores for pontine and near-normal range represented mainly by patients with pure cerebellar syndrome and one in the pathological range represented mainly by medullar atrophy had two peaks: one in the 
patients with additional non-cerebellar symptoms. CT examination of the remaining seven patients yielded closely corresponding results so that a detailed analysis of CTs is not reported.

\section{Discussion}

There is no general agreement on the nosology of the cerebellar atrophies and the multiple system atrophies which involve the cerebellum. ${ }^{1022}{ }^{26-28}$ All attempts to bring order out of the chaos of different classifications will remain difficult as long as the underlying genetical and biochemical deficits have not been identified. Following the proposal by Harding $^{29}$ to define subgroups of patients within the broad field of multiple system atrophies on the basis of their clinical presentation, the main criterion to enter into this study was a purely clinical one: cerebellar ataxia of unknown origin developing without preceding neurological symptoms after the age of 20 years. CA and OPCA are thought to be the main neuropathological categories underlying idiopathic late onset cerebellar ataxia. Although the diagnosis is usually made by histological examination of the brain at necropsy, distinctive macroscopical characteristics differentiating between CA and OPCA have been described. ${ }^{2}$ A macroscopical shrinkage of the pons may be taken as a reliable indicator for the presence of OPCA. This is the rationale for using neuroradiological methods such as CT and in particular MRI for the in vivo distinction between CA and OPCA. On the other hand, Netsky ${ }^{26}$ took the position that degeneration of the cerebellum is part of a continuum of system atrophies and that CA represents a limited form of OPCA. Our finding that the rating scores for pontine atrophy were distributed in a bimodal way supports the view that the distinction between $C A$ and OPCA is not completely arbitrary.

\section{Clinical and neuroradiological characteristics} In our patients one subgroup was defined by the presence of a pure cerebellar syndrome after a disease duration of at least four years. Clinically the patients presented with marked ataxia of gait and stance, upper limb ataxia of less degree, dysarthria and cerebellar oculomotor disturbances. All of these patients had cerebellar atrophy affecting the upper vermis, lower vermis and hemispheres to roughly the same degree with no or minor involvement of the brainstem. Although MRI is only capable of showing the macroscopical aspect of the brain, there are good reasons to assume that these patients correspond to those first described in the French literature by Thomas, ${ }^{30}$ Archambault ${ }^{31}$ and Marie et al ${ }^{9}$ and labelled originally as parenchymatous, lamellar or late cerebellar atrophy. We would prefer to use the more neutral term cortical cerebellar atrophy (CA). In contrast to the prototype cases of Marie et al, ${ }^{9}$ in which atrophy was confined to the upper vermis and anterior parts of the cerebellum, MRI in our cases showed a more diffuse atrophy confirming Netsky's ${ }^{26}$ view that there is no exclusive atrophy of the upper vermis in patients with idiopathic atrophy of the cerebellum.

It is widely believed that CA is a disease of advanced age. This view is expressed in the labelling of this neuropathological entity as late ${ }^{9}$ or senescent. ${ }^{12}$ Accordingly, the patients of Hardings's ${ }^{1}$ group A were about ten years older at the onset of symptoms than those of group C. Surprisingly, we did not find any systematic difference in the age of onset between the groups. The reasons for this are not entirely clear. It is possible that the obvious rarity of this clinical condition leads to a nonrepresentative selection of patients.

The second group of this study comprised patients who developed additional noncerebellar symptoms in the course of their disease. Clinical symptomatology, age of onset and disease progression was surprisingly homogenous. All presented with additional Parkinsonian symptoms. The occurrence of bulbar symptoms was frequent, whereas dementia, bladder dysfunction, spasticity and vertical gaze paresis were observed only in a minority of patients. Symptoms described in families with autosomal-dominant cerebellar ataxia, such as retinal pigmentary degeneration, optic atrophy, slow saccades and amyotrophy were not found in our patients. ${ }^{22} 2832 \mathrm{In}$ comparison with the patients with a pure cerebellar syndrome their clinical state was worse and they had stronger evidence for an involvement of the cerebellar hemispheres. With the exception of two, these patients had cerebellar atrophy pronounced in the cerebellar hemispheres in combination with brainstem atrophy and enlargement of the fourth ventricle. Their clinical presentation and the macroscopical aspect of their infratentorial structures correspond to those described in the classic studies by Dejerine and Thomas ${ }^{10}$ and Rosenhagen, ${ }^{4}$ in which necropsy revealed OPCA.

There were, however, exceptional patients presenting clinically with cerebellar ataxia and Parkinsonism or other non-cerebellar symptoms, in whom MRI gave no evidence of macroscopical changes in the brainstem. It is not possible to define precisely the neuropathological process during life in cases like these. Zülch ${ }^{12}$ collected a number of similar cases with a pure cerebellar atrophy at necropsy which had cerebellar ataxia and extrapyramidal increase of muscle tone during life. More recently, Adam et $a l^{33}$ found striato-nigral degeneration in patients with combinations of Parkinsonian and cerebellar symptoms. Pontine atrophy did not belong to the typical neuropathological picture.

\section{Prognostic factors}

As shown in the retrospective analysis of the patients with a longer disease duration the clinical presentation of all patients is rather uniform at the initial clinical examination. It therefore appears impossible to define clinical criteria of prognostic relevance at the time of disease onset. The only reliable prognostic marker was the latency until the first non- 
cerebellar symptom appeared. Prognosis was worse in patients who had additional symptoms from the very beginning of their disease and was better the later non-cerebellar symptoms appeared. In general, the progression rate was considerably smaller in the group of patients who did not develop non-cerebellar symptoms compared with the group with additional symptoms. Correspondingly, life-table analysis revealed a much higher mortality in patients with additional non-cerebellar symptoms. In contrast to patients with autosomal-dominant cerebellar ataxia, who experience a more rapid progression the earlier ataxia occurs, ${ }^{24}$ we find that in patients with idiopathic ataxia the age of onset does not determine prognosis.

\section{Diagnostic criteria}

Studying the clinical course of patients with idiopathic late onset cerebellar ataxia and using MRI as a means of visualising cerebellum and brainstem during life we arrived at the following conclusions: 1) A clinical differentiation between different subgroups is not possible earlier than four years after the onset of symptoms because clinical presentation tends to be uniform at the onset of symptoms; 2) The diagnosis of CA seems justified in patients with a pure cerebellar syndrome, a disease duration of at least four years and cerebellar atrophy without brainstem atrophy in MRI. MRI may, however, show pure cerebellar atrophy in exceptional patients with clinical evidence of multiple system atrophy. It is suspected that these patients have microscopical changes in other brain regions which are not revealed by MRI; 3) The diagnosis of OPCA seems justified in patients with a cerebellar syndrome and additional Parkinsonian symptoms presenting in MRI with cerebellar atrophy in combination with brainstem atrophy. It is a matter of dispute if these patients should be labelled clinically as OPCA, because the morphological picture of OPCA may be found in clinical conditions different from late onset cerebellar ataxia, such as atypical Parkinsonism or progressive supranuclear palsy; 4) A smaller number of patients suffering from idiopathic late onset cerebellar ataxia with additional noncerebellar symptoms cannot be categorised under the label of CA or OPCA. In these patients a multiple system atrophy different from OPCA must be suspected.

1 Harding AE. "Idiopathic" late onset cerebellar ataxia. A clinical and genetic study of 36 cases. J Neurol Sci 1981;51:259-71.

2 Greenfield JG. The Spino-Cerebellar Degenerations. Springfield: Charles C Thomas 1954

3 Mancall EL. Late (acquired) cortical cerebellar atrophy. In: Vinken PJ, Bruyn GW, eds. Handbook of Clinical Neurology, Vol 22. Amsterdam: Elsevier, 1975:477-508. 4 Rosenhagen H. Die primäre Atrophie des Brückenfußes und der unteren Oliven (dargestellt nach klinischen und anatomischen Beobachtungen). Arch Psychiat Nervenkr 1943;116:163-228.

5 Jellinger $K$, Tarnowska-Dziduszko E. Die ZNSVeränderungen bei den olivo-ponto-cerebellären Atrophien. Z Neurol 1971;199:192-214.

6 Berciano J. Olivopontocerebellar atrophy. A review of 117 cases. J Neurol Sci 1982;53:253-72.

7 Koeppen AH, Barron KD. The neuropathology of olivopontocerebellar atrophy. In: Duvoisin RC, Plaitakis A, eds. The Olivopontocerebellar Atrophies. New York: Raven eds. The Olivopontocer

8 Holmes G. A form of familial degeneration of the cerebellum. Brain 1907;30:466-89.

9 Marie P, Foix C, Alajouanine T. De l'atrophie cérébelleuse tardive a prédominance corticale. Rev Neurol 1922;38:849-85.

10 Dejerine J, Thomas A. L'atrophie olivo-ponto-cérébelleuse. Nouvelle Iconographie de la Salpetrière 1900;13:330-70.

1 Oppenheimer DR. Diseases of the basal ganglia, cerebellum and motor neurons. In: Blackwood W, Corsellis JAN, eds. Greenfield's Neuropathology. Chicago: Year Book Medical, 1976:608-51.

12 Zülch K-J. Úbes die primäre Kleinhirnrindenatrophie. Z Gesamte Neurol Psychiat 1936;156:493-573.

13 Eadie MJ. Cerebello-olivary atrophy (Holmes type). In: Vinken PJ, Bruyn GW, eds. Handbook of Clinical Neurology, Vol 22. Amsterdam: Elsevier, 1975:403-14.

14 Destunis G. Die olivo-ponto-cerebellare Heredoataxie. Z Gesamte Neurol Psych 1944;177:683-99.

15 Carter HR, Sukavajana C. Familial cerebello-olivary degeneration with late development of rigidity and dementia. Neurology 1956;6:876-84.

16 Gilroy J, Lynn GE. Computerized tomography and auditory-evoked potentials. Arch Neurol 1978;35:143-7.

7 Allen JH, Martin JT, McLain LW. Computed tomography in cerebellar atrophic processes. Neuroradiology 1979;130:379-82.

18 Koller WC, Glatt SL, Perlik S, Huckman MS, Fox JH Cerebellar atrophy demonstrated by computed tomoCerebellar atrophy demonstrated

19 Huang YP, Plaitakis A. Morphological changes of olivopontocerebellar atrophy in computed tomography and comments on its pathogenesis. In: Duvoisin RC, Plaitakis A, eds. The Olivopontocerebellar Atrophies. New York: Raven Press, 1984:39-85.

20 Garcia de la Rocha ML, Moreno Martinez JM, Garrido Carrion A, Margalet Fernández P, Martin Araguz A. Present criteria for the diagnosis in vivo of olivopontocerebellar atrophy. Acta Neurol Scand 1988;77: 234-8.

21 Nabatame H, Fukuyama $H$, Akiguchi I, Kameyama $M$, Nishimura K, Nakano Y. Spinocerebellar degeneration: Qualitative and quantitative MR analysis of atrophy. $J$ Comp Assist Tomography 1988;12:298-303.

22 Harding AE. The clinical features and classification of the late onset autosomal dominant cerebellar ataxias. A study of 11 families, including descendants of "The Drew Family of Walworth". Brain 1982;105:1-28.

23 Harding AE. Classification of the hereditary ataxias and paraplegias. The Lancet 1983;i:1151-5.

24 Currier RD, Jackson JF, Meydrech EF. Progression rate and age at onset are related in autosomal dominant neurologic diseases. Neurology 1982;32:907-9.

25 Kaplan EL, Meier P. Nonparametric estimation from incomplete observations. J Am Stat Ass 1958;53:457-81.

26 Netsky MG. Degenerations of the cerebellum and its pathways. In: Minckler J, ed. Pathology of the Nervous System. New York: McGraw-Hill, 1968:1163-85.

27 Eadie MJ. Olivo-ponto-cerebellar atrophy (DejerineThomas type). In: Vinken PJ, Bruyn GW, eds. Handbook of Clinical Neurology, Vol 22. Amsterdam: Elsevier, 1975:415-31.

28 Eadie MJ. Olivo-ponto-cerebellar atrophy (Menzel type). In: Vinken PJ, Bruyn GW, eds. Handbook of Clinical In: Vinken PJ, Bruyn GW, eds. Handbook of Clinical

29 Harding AE. Commentary: Olivopontocerebellar atrophy is not a useful concept. In: Marsden CD, Fahn S, eds. Movement Disorders 2. London: Butterworth, 1987: 269-71.

30 Thomas A. Atrophie lamellaire des cellules de Purkinje. Rev Neurol 1905;13:917-24.

31 Archambault, La Salle. Parenchymatous atrophy of the cerebellum. J Nerv Ment Dis 1918;48:273-312.

32 Coutinho $\mathrm{P}$, Andrade C. Autosomal dominant system degeneration in Portuguese families of the Azores Islands. Neurology 1978;28:703-9.

33 Adams RD, Salam-Adams M. Striatonigral degeneration. In: Vinken PJ, Bruyn GW, Klawans HL, eds. Handbook of Clinical Neurology, Vol 5. Amsterdam: Elsevier, 1986: 205-12. 